\title{
A PITFALL TRAPPING SURVEY OF BEETLES IN STEPPE ECOSYSTEMS OF THE REPUBLIC OF MOLDOVA
}

\author{
Natalia Munteanu-Molotievskiy, Anna Moldovan, Ion Toderas \\ Institute of Zoology, Chisinau, Republic of Moldova, \\ e-mail: munteanu_natalia_v@yahoo.com
}

\begin{abstract}
A pitfall survey of beetles in steppe ecosystems of the Republic of Moldova was conducted. Five locations were investigated, two from the Balti steppe and three from the Bugeac steppe. As a result, a total of 563 beetle specimens, belonging to 98 species, 51 genera, and 15 families were collected. Among families recorded Carabidae was the one with the highest number of species. The ecological features of collected beetle species were revealed. The results show that steppes are still important biodiversity reservoirs within the boundaries of the country and urgent conservation activities are required.
\end{abstract}

\section{Introduction}

The biodiversity of a region is mainly determined by three factors: climate, geology, and evolutionary processes that occur in that territory. The influence of these factors and their relationship leads to the establishment of a territorial formation or unit called today by scientists as ,ecoregions” (Dinerstein et al. 2001). Now there are 142 terrestrial, 53 freshwater and 43 marine ecoregions recognized across the globe.

The Republic of Moldova is a small landlocked country situated in south-eastern Europe between Ukraine and Romania. Vegetal coating and mottled landscapes composed of grassland interspersed with areas of woodland are defined by the unique positioning of the country at the crossroad of three terrestrial ecoregions Central European mixed forests, East European Forest steppe, and Pontic-Caspian steppe.

The Central European mixed forest ecoregion extends from eastern Germany to the northern part of the Republic of Moldova and north-eastern Romania. A distinct aspect of this ecoregion is the presence of thermophilous plant species common for warmer and drier habitats, particularly in the south-eastern part of the ecoregion, which borders the forest-steppe zone of Ukraine, the Republic of Moldova, and Romania.

The East European Forest steppe ecoregion is a patchwork of broadleaf forest stands and grasslands (steppe) that stretches across eastern Europe from the Ural Mountains through Central Russia to the middle of Ukraine, isolated areas of a similar character being found in eastern Romania, Republic of Moldova, and Bulgaria.

The Pontic-Caspian steppe ecoregion stretches from the northern shores of the Black Sea to the northern area around the Caspian Sea. It starts from Dobruja in the north-eastern corner of Bulgaria and south-eastern Romania and continues through the Republic of Moldova and southern and eastern Ukraine, across the Russian Northern Caucasus, the Southern and lower Volga regions to western Kazakhstan, both forming part of the larger Eurasian Steppe (www. worldwildlife.org).

In the Republic of Moldova, the steppe ecosystems are situated in the northern part of the 
country, the Balti steppe, with a total surface of $1920 \mathrm{~km}^{2}$, and in the southern part, the Bugeac steppe covering a surface of $3210 \mathrm{~km}^{2}$, divided by Codrii forest in the middle.

Specific for Bugeac steppe are primary vegetation communities consisting of feather grass (Stipa capilata L., S. lessingiana Trin. et Rupr., S. ucrainica P. Smirn.), and fescue grass (Festuca valesiaca Gandin), degraded or secondary steppe sectors comprising yellow bluestem (Botriochloa ischaemum (L.) Keng), bluegrass (Poa angustifolia L.), and couch grass (Elytrigia repens (L.) Nevski). The phytocenoses of the Balti steppe are mainly composed of feather grass (Stipa pulcherrima C. Koch, S. lessingiana Trin. et Rupr.), and are spread over a third of the territory, less common are formations of fescue grass (Festuca valesiaca Gandin), while the sunnier slopes prone to landslides are usually suitable for yellow bluestem (Botriochloa ischaemum (L.) Keng) (Lazu, Teleuţă \& Chirtoacă, 2006).

Grasslands are considered regional biodiversity hotspots and consequently of high conservation value. Unfortunately, the steppe is one of the most transformed and overworked ecosystems, and at the same time the most underrated of landscapes. In ecosystem processes, insects have an important role as pollinators, food chain elements, maintenance, and improvement of soil quality. Being sensitive to the changes of local resources, they are good indicators at the landscape level. Insects are of increasing interest to conservation practitioners, among which beetles have been widely proposed as a model for biodiversity inventory and monitoring, as they satisfy all the criteria necessary in ecological research and biodiversity survey (Eyre \& Luff, 1990, Halffter \& Favila, 1993, Ohsawa, 2010).

Despite the conservation value of the steppe-like grassland of the Republic of Moldova, little is known about the beetle diversity and community structure. This study aims to survey the fauna of beetles in steppe ecosystems.

\section{Material and methods}

The study presented was conducted in steppe ecosystems of the Republic of Moldova. Field trips took place between August and October 2015, sampling covered two sites from Balti steppe: Pelinia $\left(47.8780^{\circ} \mathrm{N}, 27.8344^{\circ} \mathrm{E}\right)$ and Vranesti $\left(47.6233^{\circ} \mathrm{N}, 28.1009^{\circ} \mathrm{E}\right)$ and three from Bugeac steppe: Stefanesti $\left(46.4459^{\circ} \mathrm{N}, 29.6892^{\circ} \mathrm{E}\right)$, Bugeac $\left(46.3658^{\circ} \mathrm{N}, 28.6633^{\circ} \mathrm{E}\right)$, and $\mathrm{Ci}-$ umai $\left(45.7822^{\circ} \mathrm{N}, 28.5697^{\circ} \mathrm{E}\right)$.

Specimens were captured out using pitfall traps. By ten traps were placed on each of the investigated sites, samples were collected every seven to ten days, due to unforeseeable circumstances. Traps were made of a $700 \mathrm{~cm} 3$ jar, set up at the ground level, and filled with saturated $\mathrm{NaCl}$ solution. Recovered specimens were transported to the laboratory for further counting and identification.

Taxonomic identification of collected beetles was carried out using Keys to Insects of the European Part of the USSR, vol. 2 (1965), and some additional online resources (Mike's Insect Keys and Käfer Europas).

\section{Results and discussions}

As a result of the survey from five localities, a total of 563 beetle specimens, belonging to 
98 species, 51 genera, and 15 families were caught (table below). Among families recorded Carabidae was the one with the highest number of species (51), followed by Curculionidae (9), Scarabaeidae (6), Tenebrionidae and Dermestidae (by 5 species), Histeridae, and Chrysomelidae (by 4), Staphylinidae and Silphidae (by 3), Cerambycidae and Trogidae (by 2), and Buprestidae, Endomychidae, Cryptophagidae and Coccinellidae - by one species each. Of all collected beetles, ground beetles (Carabidae) represented the greatest proportion in terms of the number of individuals (368 specimens), followed by Tenebrionidae family with 50 specimens, Staphylinidae (37 specimens), Curculionidae (30), Dermestidae (22), Trogidae (16), Histeridae (12), Scarabaeidae (11), Silphidae and Chrysomelidae (by 5), Cerambycidae and Buprestidae (by 2) and Endomychidae, Cryptophagidae and Coccinellidae - by one specimen.

Table. Taxonomic composition and ecological features of beetle species collected

\begin{tabular}{|c|c|c|c|c|c|c|c|c|}
\hline \multirow{3}{*}{ Taxa } & \multicolumn{5}{|c|}{ Samples } & \multirow{3}{*}{ 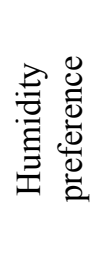 } & \multirow{3}{*}{ 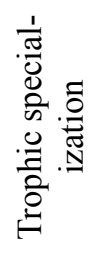 } & \multirow{3}{*}{ 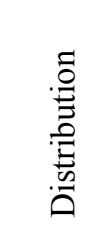 } \\
\hline & \multicolumn{2}{|c|}{ Balti } & \multicolumn{3}{|c|}{ Bugeac } & & & \\
\hline & $\frac{\pi}{. \Xi}$ & 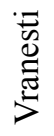 & 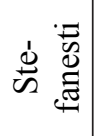 & $\begin{array}{l}\mathscr{J} \\
\mathbb{\Xi} \\
\stackrel{0}{\Xi}\end{array}$ & 䇂 & & & \\
\hline \multicolumn{9}{|l|}{ Carabidae } \\
\hline Acinopus ammophilus Dej. & & & & & 2 & MzX & Omn & E-Asn \\
\hline A. laevigatus Mén. & & & 1 & & & Mzx & Omn & E-Asn \\
\hline A. picipes (O1.) & & & 2 & & 1 & Mzx & Omn & E-Med \\
\hline Amara aenea $\mathrm{DeG}$. & & & & & 1 & $\mathrm{Xr}$ & $\mathrm{Ph}$ & T-Pal \\
\hline A. brunnea Gyll. & & & 1 & & & Ms & Omn & Ola \\
\hline A. consularis (Duft.) & 1 & & & & & Mzx & Omn & $\mathrm{E}-\mathrm{Si}$ \\
\hline A. equestris (Duft.) & 2 & & & & & Mzx & $\mathrm{Omn}$ & E-Si \\
\hline A. littorea Thoms. & 1 & & & & & Ms & Omn & Eur \\
\hline A. sabulosa (Serville) & 1 & & & & & Ms & Omn & Pal \\
\hline Brachinus crepitans (L.) & & 1 & 1 & 14 & 1 & Mzx & $\mathrm{Z}$ & W-Pal \\
\hline Calathus ambiguus (Payk.) & 1 & & & 1 & & Mzx & $\mathrm{Z}$ & E-Asn \\
\hline C. distinguendus Chaud. & & & & & 1 & Mzx & $\mathrm{Z}$ & Med \\
\hline C. erratus (Sahlb.) & 1 & & & & & Mzx & $\mathrm{Z}$ & E-Si \\
\hline C. fuscipes (Goeze) & 51 & & 12 & 7 & & Ms & Omn & W-Pal \\
\hline C. melanocephalus (L.) & & & & 2 & & Mzx & $\mathrm{Z}$ & T-Pal \\
\hline Carabus nemoralis Müller & & & 1 & & & Ms & $\mathrm{Z}$ & Eur \\
\hline $\begin{array}{l}\text { Cryptophonus tenebrosus } \\
\text { (Dej.) }\end{array}$ & 1 & & & & 3 & Ms & Omn & E-Med \\
\hline Cymindis axillaris (F.) & & & & 1 & & $\mathrm{Xr}$ & $\mathrm{Z}$ & W-Pal \\
\hline Dolichus halensis (Schall.) & & & & & 1 & Ms & $\mathrm{Z}$ & T-Pal \\
\hline
\end{tabular}




\begin{tabular}{|c|c|c|c|c|c|c|c|c|}
\hline Harpalus atratus Latr. & 1 & 1 & & & & Msh & Omn & Pal \\
\hline H. cephalotes Fairm., Laboul. & & & 1 & & 1 & $\mathrm{Hl}$ & $\mathrm{Ph}$ & Eur \\
\hline H. dimidiatus (P. Rossi) & & 2 & & & & $\mathrm{Xr}$ & Omn & E-Asn \\
\hline H. dispar splendens Gebl. & & & & & 6 & $\mathrm{Hl}$ & Omn & W-Pal \\
\hline H. distinguendus (Duft.) & & & 1 & & & Ms & Omn & T-Pal \\
\hline H. hospes Sturm & & 14 & & 26 & & $\mathrm{Hl}$ & Omn & T-Pal \\
\hline H. melancholicus Dej. & & 2 & & 4 & 4 & $\mathrm{Xr}$ & Omn & Pal \\
\hline H. modestus Dej. & & & & 1 & & Ms & Z & E-Asn \\
\hline H. politus Dej. & & 2 & & & & Msh & Omn & W-Pal \\
\hline H. pygmaeus Dej. & 16 & & 1 & 4 & & $\mathrm{Xr}$ & Omn & E-Med \\
\hline H. rubripes Duft. & & 1 & & & & Ms & Omn & T-Pal \\
\hline H. serripes (Quens.) & & & & 1 & & Mzx & Omn & E-Med \\
\hline H. smaragdinus (Duft.) & & & & & 1 & Ms & Omn & E-Asn \\
\hline H. tardus (Panz.) & & 3 & & 1 & 1 & Ms & Omn & E-Asn \\
\hline Laemostenus terricola Hbst. & & & 5 & 1 & & Msh & $\mathrm{Z}$ & E-C \\
\hline Licinus cassideus (F.) & & & 4 & & & Ms & $\mathrm{Z}$ & E-Med \\
\hline Ophonus azureus (F.) & 2 & 4 & 4 & 1 & 12 & Mzx & Omn & E-Med \\
\hline O. convexicollis Men. & & & & & 1 & Mzx & Omn & E-Med \\
\hline O. diffinis (Dej.) & & 2 & & & & Ms & $\mathrm{Ph}$ & E-Med \\
\hline O. laticollis Mnrhm. & & 2 & & & & $\mathrm{Xr}$ & $\mathrm{Ph}$ & Pal \\
\hline O. rufibarbis (F.) & 1 & & 8 & 1 & 6 & $\mathrm{Xr}$ & $\mathrm{Ph}$ & Pal \\
\hline O. sabulicola (Panz.) & 1 & 4 & 6 & & 1 & Ms & Omn & E-Med \\
\hline Paradromius linearis (Ol.) & 1 & & & & 1 & Ms & $\mathrm{Z}$ & E-Med \\
\hline Parophonus hirsutulus (Dej.) & & 1 & & & & $\mathrm{Hg}$ & $\mathrm{Z}$ & T-Med \\
\hline $\begin{array}{l}\text { Pseudoophonus griseus } \\
\text { (Panz.) }\end{array}$ & 1 & 1 & 3 & & & Ms & Omn & T-Pal \\
\hline P. rufipes (DeG.) & 8 & 1 & 28 & 1 & 1 & Ms & Omn & E-Si \\
\hline Pterostichus macer (Marsh.) & 1 & 2 & & 1 & & Ms & $\mathrm{Z}$ & E-Asn \\
\hline P. melas (Creutz.) & & & & 9 & & Ms & Omn & E-Med \\
\hline Sphodrus leucophthalmus (L.) & & & & & 1 & Msh & $\mathrm{Z}$ & W-Pal \\
\hline $\begin{array}{l}\text { Trechus quadristriatus } \\
\text { (Shrnk.) }\end{array}$ & 1 & & & 1 & & Ms & $\mathrm{Z}$ & E-Asn \\
\hline Zabrus spinipes (F.) & & & & 10 & 1 & Ms & Omn & E-Med \\
\hline Z. tenebrioides (Goeze) & 18 & & 2 & & & Ms & Omn & E-Med \\
\hline \multicolumn{9}{|l|}{ Histeridae } \\
\hline $\begin{array}{l}\text { Gnathoncus disjunctus su- } \\
\text { turifer } \text { Rtt. }\end{array}$ & & & & 2 & & Mzx & Omn & Eur \\
\hline Hister quadrimaculatus L. & & & & 1 & & Ms & $\mathrm{N}$ & E-Asn \\
\hline
\end{tabular}




\begin{tabular}{|c|c|c|c|c|c|c|c|c|}
\hline Saprinus maculatus (Rossi) & 1 & & & & 1 & Mzx & $\mathrm{N}$ & E-Asn \\
\hline S. semistriatus (Scriba) & & & & 5 & 2 & Mzx & $\mathrm{N}$ & E-Si \\
\hline \multicolumn{9}{|l|}{ Silphidae } \\
\hline Nicrophorus antennatus (Rtt.) & 1 & & & & & $\mathrm{Xr}$ & $\mathrm{N}$ & E-Si \\
\hline N. germanicus (L.) & & & & 1 & 1 & $\mathrm{Xr}$ & $\mathrm{N}$ & Pal \\
\hline N. interruptus Steph. & & & & & 2 & $\mathrm{Xr}$ & $\mathrm{N}$ & T-Pal \\
\hline \multicolumn{9}{|l|}{ Staphylinidae } \\
\hline Lathrobium brunnipes (F.) & 20 & 2 & & & & $\mathrm{Hg}$ & $\mathrm{Z}, \mathrm{p}$ & T-Pal \\
\hline Ocypus ophthalmicus (Scop.) & & & 8 & & & $\mathrm{Xr}$ & $\mathrm{Z}, \mathrm{p}$ & Pal \\
\hline Platydracus stercorarius (Ol.) & & & 7 & & & $\mathrm{Xr}$ & $\mathrm{Z}$ & Pal \\
\hline \multicolumn{9}{|l|}{ Trogidae } \\
\hline Trox hispidus Pontop. & & 3 & & 3 & 7 & $\mathrm{Xr}$ & $\mathrm{N}$ & Eur \\
\hline T. sabulosus (L.) & 2 & & & 1 & & $\mathrm{Xr}$ & $\mathrm{N}$ & Eur \\
\hline \multicolumn{9}{|l|}{ Scarabaeidae } \\
\hline Onthophagus furcatus $\mathrm{F}$. & & & & 4 & & $\mathrm{Xr}$ & $\mathrm{C}$ & T-E-Med \\
\hline O. ovatus (L.) & & & & & 2 & $\mathrm{Xr}$ & $\mathrm{C}$ & Pal \\
\hline O. ruficapillus Brulle & & & & 1 & & $\mathrm{Xr}$ & $\mathrm{C}$ & Pal \\
\hline O. semicornis (Panz.) & 2 & & & & & $\mathrm{Xr}$ & $\mathrm{C}$ & Pal \\
\hline Pentodon idiota (Hbst.) & 1 & & & & & $\mathrm{Xr}$ & $\mathrm{Ph}$ & E-Med \\
\hline Sisyphus schaefferi (L.) & & & & 1 & & $\mathrm{Xr}$ & $\mathrm{C}$ & Eur \\
\hline \multicolumn{9}{|l|}{ Buprestidae } \\
\hline Trachys fragariae Bris. & & & 2 & & & $\mathrm{Xr}$ & $\mathrm{Ph}$ & Eur \\
\hline \multicolumn{9}{|l|}{ Dermestidae } \\
\hline $\begin{array}{l}\text { *Dermestes intermedius } \\
\text { Kalik }\end{array}$ & & & & 1 & & $\mathrm{Xr}$ & $\mathrm{N}$ & Eur \\
\hline D. laniarius Ill. & 2 & 1 & 5 & 1 & 2 & $\mathrm{Xr}$ & $\mathrm{N}$ & Pal \\
\hline D. maculatus DeG. & & & & & 1 & $\mathrm{Xr}$ & $\mathrm{N}$ & $\operatorname{Cos}$ \\
\hline D. mustelinus Er. & & & & & 8 & $\mathrm{Xr}$ & $\mathrm{N}$ & Pal \\
\hline D. undulatus Brahm & & & & & 1 & $\mathrm{Xr}$ & $\mathrm{N}$ & Ola \\
\hline \multicolumn{9}{|l|}{ Tenebrionidae } \\
\hline Asida lutosa Solier & & & & 1 & & $\mathrm{Xr}$ & $\mathrm{Ph}$ & S-E Eur \\
\hline Crypticus quisquilius (L.) & 2 & & 6 & 8 & 2 & $\mathrm{Xr}$ & $\mathrm{Ph}$ & E-Si \\
\hline Oodescelis polita (Sturm) & & & 5 & & & $\mathrm{Xr}$ & $\mathrm{Ph}$ & Med \\
\hline Opatrum sabulosum (L.) & & & 1 & 5 & 17 & $\mathrm{Xr}$ & $\mathrm{Ph}$ & E-Si \\
\hline Pedinus femoralis (L.) & & & 3 & & & $\mathrm{Xr}$ & $\mathrm{Ph}$ & P-Med \\
\hline \multicolumn{9}{|l|}{ Endomychidae } \\
\hline Lycoperdina succincta (L.) & & & & 1 & & Msh & $\mathrm{F}$ & Pal \\
\hline Cryptophagidae & & & & & & & & \\
\hline
\end{tabular}




\begin{tabular}{|c|c|c|c|c|c|c|c|c|}
\hline Cryptophagus pilosus Gyll. & & & & 1 & & Mzx & $\mathrm{F}$ & Ola \\
\hline \multicolumn{9}{|l|}{ Coccinellidae } \\
\hline $\begin{array}{l}\text { Hyperaspis campestris } \\
\text { (Hbst.) }\end{array}$ & & & & & 1 & $\mathrm{Xr}$ & $\mathrm{Z}$ & Eur \\
\hline \multicolumn{9}{|l|}{ Cerambycidae } \\
\hline Dorcadion fulvum (Scop.) & & & & 1 & & Mzx & $\mathrm{Ph}$ & Eur \\
\hline $\begin{array}{l}\text { Neodorcadion bilineatum } \\
\text { (Germ.) }\end{array}$ & & & & & 1 & Mzx & $\mathrm{Ph}$ & Med \\
\hline \multicolumn{9}{|l|}{ Chrysomelidae } \\
\hline Chrysolina haemoptera (L.) & & 1 & & & & $\mathrm{Xr}$ & $\mathrm{Ph}$ & Pal \\
\hline Ch. limbata (F.) & & 2 & & & & Mzx & $\mathrm{Ph}$ & Pal \\
\hline $\begin{array}{l}\text { Coptocephala unifasciata } \\
\text { (Scop.) }\end{array}$ & & & & & 1 & Mzx & $\mathrm{Ph}$ & W-Pal \\
\hline Galeruca tanaceti (L.) & 1 & & & & & Mzx & $\mathrm{Ph}$ & Ola \\
\hline \multicolumn{9}{|l|}{ Curculionidae } \\
\hline Archeophloeus inermis (Boh.) & & & & 1 & & $\mathrm{Xr}$ & $\mathrm{Ph}$ & Pal \\
\hline Cleonis pigra (Scop.) & 2 & & 4 & & & Mzx & $\mathrm{Ph}$ & Ola \\
\hline Otiorhynchus fullo (Schrnk.) & & & & 7 & & Ms & $\mathrm{Ph}$ & Eur \\
\hline O. ligustici (L.) & & & & & 7 & Ms & $\mathrm{Ph}$ & Ola \\
\hline O. ovatus (L.) & & & & 1 & 3 & Ms & $\mathrm{Ph}$ & Ola \\
\hline $\begin{array}{l}\text { Pseudocleonus cinereus } \\
\text { (Schrnk.) }\end{array}$ & & 1 & & & & Mzx & $\mathrm{Ph}$ & W-Pal \\
\hline Sitona lateralis Gyll. & & & & 1 & & Mzx & $\mathrm{Ph}$ & Eur \\
\hline S. suturalis Steph. & & & & 2 & & Mzx & $\mathrm{Ph}$ & T-Pal \\
\hline $\begin{array}{l}\text { Trachyphloeus alternans } \\
\text { (Gyll.) }\end{array}$ & & & & & 1 & Ms & $\mathrm{Ph}$ & Eur \\
\hline
\end{tabular}

Humidity preference: Hg - hygrophilous, HI - halophilous, Msh - mesohydrophilous, Xr - xerophilous, Ms - mesophilous, Mzx - mesoxerophilous; Trophic specialization: Omn - Omnivorous (carnivorous + partly granivorous in case of Carabidae, carnivorous + partly fungivorous in case of Histeridae), $\mathbf{F}$ - fungivorous, $\mathbf{P h}$ - Phytophagous, $\mathbf{Z}$ - Zoophagous ( $\mathrm{p}$ - predators), N - Necrophagous, C - coprophagous. Distribution: Ola - Holarctic, Pal - Palaearctic, W-Pal - West-Palaearctic, T-Pal - TransPalaearctic, Eur - European, E-C - Euro-Caucasian, E-Si - Euro-Siberian, E-Asn - Euro-Asian, C-Asn - Central-Asian, S-E Eur - South-East European, E-Med - Euro-Mediterranean, T-E-Med - Turanic-Euro-Mediterranean, T-Med - Turanic-Mediterranean, Med - Mediterranean, P-Med - Ponto-Mediterranean, Cos - Cosmopolitan. *First record of the species for RM fauna.

According to hygropreference the typical xerophilous species were represented by 34 species, followed by mesophilous (28), mesoxerophilous (26); mesohydrophilous (5), halophilous (3), and hygrophilous (2).

By trophic specialization, six groups were distinguished, of which omnivorous counted 31 species, followed by phytophagous (27), zoophagous (20), necrophagous (13), coprophagous (5), and fungivorous (2).

Giving their current distribution, the 98 identified species from all five investigated sites can be classified into 15 zoogeographical categories: Palaearctic (17 species), European (14), Eu- 
ro-Mediterranean (14), Euro-Asian (11), Trans-Palaearctic (10), West-Palaearctic (8), Euro-Siberian (8), Holarctic (7), Mediterranean (3), Euro-Caucasian, South-East European, Turanic-Euro-Mediterranean, Turanic-Mediterranean, Ponto-Mediterranean, and Cosmopolitan by one species.

The natural ecosystems of the Republic of Moldova are highly degraded due to human impact. Today, in the Republic of Moldova, nearly two-thirds of the territory is used for agricultural purposes, with natural ecosystems covering only about $15 \%$ of the country. Of that, native steppe, and steppe-associated wet meadows are almost inexistent, making around 1.9\%, those are usually areas unsuitable for agriculture (Biodiversity Assessment for Moldova, 2001, Postolache \& Ghendov, 2020). Around 1200 areas, within 1 to 300 ha, with steppe and meadow vegetation, are known to be preserved in small, isolated patches, and most of them are being used as pastures (Postolache, 1995).

Over the years much of steppe ecosystems have been converted into arable land for intensive agriculture, suffering from soil depletion and degradation (Mordkovich, 2014). Therefore, steppes have been identified as a major conservation priority for both biodiversity value and landscapes. This is a first attempt to uncover the beetle diversity in the steppe ecosystems of the Republic of Moldova. Obtained results confirmed our expectation that steppes are still important biodiversity reservoirs within the boundaries of our country. There is an urgent need towards rising attention of the local and central public authorities, and population on the priority of the biodiversity conservation measures in the steppe region. The most important areas from steppe ecosystems of the Republic of Moldova must be identified and conserved as natural reserves within the National Ecological Network. Future investigations should focus on extending the number of sampling localities for better coverage of the steppe ecosystems of the Republic of Moldova, and the application of additional sampling methods to extend the range of insect groups.

Acknowledgments. These studies were financially supported by Rufford Small Grant, The Rufford Foundation, and partially by institutional project (2014-2019) 5.817.02.12F, and State Program (20202023) project 20.80009.7007.12.

\section{Bibliography}

1. Biodiversity Assessment for Moldova. Task Order under the Biodiversity and Sustainable Forestry IQC(BIOFOR). USAID/Kiev, Ukraine, 2001. 52 p.

2. Dinerstein E., Wikramanayake E., Burgess N., Powell G., Underwood E.C., D'Amico J., et al. 2001. Terrestrial ecoregions of the world: a new map of life on earth. In: BioScience, 51(11): 933-938.

3. Eyre M.D., Luff M.L. 1990. A preliminary classification of European grassland habitats using carabid beetles. In: Stork N.E. (ed.), The Role of Ground Beetles in Ecological and Environmental Studies. Intercept, Andover, UK. 227-236 p.

4. Halffter G., Favila M.E. 1993. The Scarabaeinae (Insecta: Coleoptera) an animal group for analysing, inventorying and monitoring biodiversity in tropical rainforest and modified landscapes. In: Biology International, 27, p. 15-21.

5. Keys to insects of the European part of the USSR in five volumes. Volume 2. Coleoptera and 
Strepsiptera. Ed. E.L. Guryeva and O.L. Kryzhanovsky. no. 89. Publishing "Science”. Moscow-Leningrad. 1965. 668 p. (In Russian).

6. Lazu Ş., Teleuţă A., Chirtoacă V. 2006. Pajiştile de stepă naturală şi necesitatea extinderii ariei acestora în Republica Moldova. In: Mediul Ambiant, 2(26), p. 24-26.

7. Mike's Insect Keys. Available online: https://sites.google.com/view/mikes-insect-keys/mikesinsect-keys (accessed in 2015-2016).

8. Mordkovich V.G. 2014. Steppe ecosystems. V.G. Mordkovich; ed. I.E. Smelyansky. 2nd ed. rev. and add. Novosibirsk: Academic publishing house "Geo". 170 p. (In Russian).

9. Ohsawa M. 2010. Beetle families as indicators of Coleopteran diversity in forests: a study using Malaise traps in the central mountainous region of Japan. In: Journal of Insect Conservation, 14, p. 479-484.

10. Places, Ecoregions, Terrestrial Ecoregions. World Wildlife Federation. https://www. worldwildlife.org/biome-categories/terrestrial-ecoregions (Retrieved June 23, 2021).

11. Postolache G. 1995. Vegetația Republicii Moldova. Academia de Științe a Republicii Moldova, Institutul de Botanică. Chișinău, Moldova.

12. Postolache G., Ghendov V. 2020. Flora și vegetația Republicii Moldova în contextul impacturilor naturale și antropice. In: Akademos, 2, p. 22-31.

The beetles of Europe. A reference work on the Internet published by Arved Lompe, Nienburg/ Weser. http://coleonet.de/coleo/index.htm (accessed in 2015-2016). (German). 